\title{
Criminologie
}

\section{Index cumulatif 1968-1981, Vol. I-XIV}

\section{Jacqueline De Plaen}

Volume 15, numéro 1, 1982

Droit et justice

URI : https://id.erudit.org/iderudit/017155ar

DOI : https://doi.org/10.7202/017155ar

Aller au sommaire du numéro

Éditeur(s)

Les Presses de l'Université de Montréal

ISSN

0316-0041 (imprimé)

1492-1367 (numérique)

Découvrir la revue

Citer ce document

De Plaen, J. (1982). Index cumulatif 1968-1981, Vol. I-XIV. Criminologie, 15(1),

123-146. https://doi.org/10.7202/017155ar d'utilisation que vous pouvez consulter en ligne.

https://apropos.erudit.org/fr/usagers/politique-dutilisation/ 


\title{
INDEX CUMULATIF CRIMINOLOGIE
}

\author{
Préparé par: \\ Jacqueline De Plaen \\ Responsable du Service de documentation, C.I.C.C.
}




\section{INDEX DES AUTEURS ET COLLABORATEURS}

Année Vol./No. Pages

AKMAN, Doyan D.

Towards the measurement of criminality in Canada : a replication study

1968

I

$135-260$

AMESSE-GIRARD, Jocelyne

Nouvelles avenues considérées par le service de probation

1979

XII 2

$41-57$

ARCAND, Suzanne

Comparative criminology :

Africa

1973

VI

199-217

ARSENAULT, Carmen

La libération conditionnelle : le point de vue des acteurs

1981

XIV $2 \quad 41-60$

BARIL, Micheline

Violence et répression : mythe ou réalité?

Ils n'ont plus la liberté : réactions à la victimisation et ses conséquences

\begin{tabular}{|c|c|c|}
\hline 1979 & XII I & $66-77$ \\
\hline 1980 & XIII 1 & 94-103 \\
\hline 1969 & II & $71-144$ \\
\hline 1978 & XI 1 & $80-86$ \\
\hline 1978 & XI 1 & $46-64$ \\
\hline 1973 & $X V$ & $199-217$ \\
\hline 1979 & XII l & $6-23$ \\
\hline 1976 & IX 1-2 & $196-218$ \\
\hline 1980 & XIII 2 & $79-98$ \\
\hline
\end{tabular}

BERTRAND, Marie-Andrée

Self-image and delinquency : a contribution to the study of female criminality and woman's image

BLAZINA, Vesna

Bibliographie sélective sur la rééducation

BRILL, Ronald

Traitement des jeunes délinquants : modèle d'appariement selon le niveau conceptuel

BRILLON, Yves

Comparative criminology : Africa.

Le rôle et l'utilité du public dans l'élaboration des politiques criminelles

SODER, Jean-Pau

Surveiller et punir

La Crise d'octobre et les commissions d'enquête 


\section{BRUNET-AUBRY, Lise}

Littérature carcérale québécoise

CAMPEAU, André

Les prisons du Québec; ghetto des pauvres

CARRIËRE, Pierre

Le comité Paul Rose

CHARBONNEAU, Jean-Pierre

La pègre, un milieu d'affaires

CLICHE, Robert

Le crime organisé et les pactes de nonagression. Réflexions du juge Cliche recueillies par A. Parizeau

COLETTE-CARRIÈRE, Renée

La victimologie et le viol, un discours complice

The cost of crime and of social defence against crime : Summary of the Second International Symposium in Comparative Criminology

\section{CUSSON, Maurice}

Deux modalités de la peine et leurs effets sur le criminel

L'observation du comportement des jeunes en institution

Les normes de l'intervention auprès des jeunes mésadaptés - bilan des écrits

\section{CZETWERTYNSKA, Aniela}

Aperçu bibliographique des travaux de l'Université de Montréal, 19601978

DANDURAND, Yvon

La surveillance de la liberté et la pratique criminologique

DEBUYST, Christian

Vers une nouvelle conception des valeurs : apport de la psychologie à la criminologie des valeurs

La revue Criminologie et la recherche

\section{DELISLE, D.}

La prison de Bordeaux et sa population
1973

VI

$67-146$

Année

Vol./No. Pages

1976

IX 1-2

191-195

1976

IX 1-2

$60-86$

1981

XIV 2

$95-98$

1977

X 1

$29-45$

1977

X 1

69.78

1980

XIII 1

$60-79$

1971

IV

193-209

1974

VII

11-52

1975

VIII 1-2

$119-144$

1978

XI 2

42-75

1977

$\mathrm{X} 2$

93-107

1979

78.88

1977

$\mathrm{X} 2$

74-77

$1976 \quad$ IX 1-2 23-59

XII 2 


\begin{tabular}{|c|c|c|c|}
\hline & Année & Vol./No. & Pages \\
\hline $\begin{array}{l}\text { DE PLAEN, Jacqueline } \\
\text { Aperçu bibliographique des travaux } \\
\text { de l'Université de Montréal, 1960- } \\
1978\end{array}$ & 1977 & $\times 2$ & $93-107$ \\
\hline Bibliographie générale & 1979 & XII 2 & $101-105$ \\
\hline Information bibliographique & 1980 & XIII 1 & $107-108$ \\
\hline La Crise, telle qu'on l'a écrite & 1980 & XIII 2 & $99-110$ \\
\hline Information bibliographique & 1981 & XIV 1 & $111-118$ \\
\hline $\begin{array}{l}\text { Le Département de criminologie de } \\
\text { l'Université de Montréal : enseigne- } \\
\text { ment et recherches en cours }\end{array}$ & 1969 & il & $169-191$ \\
\hline $\begin{array}{l}\text { DESLAURIERS, Lise } \\
\text { Interprétation psycho-culturelles de } \\
\text { l'inadaptation juvénile dans la société } \\
\text { de masse contemporaine }\end{array}$ & 1968 & 1 & $9-134$ \\
\hline $\begin{array}{l}\text { DOUYON, Emerson } \\
\text { La transe vaudouesque : un syndrome } \\
\text { de déviance psycho-culturelle }\end{array}$ & 1969 & II & $11-70$ \\
\hline $\begin{array}{l}\text { La famille et la délinquance dans trois } \\
\text { sphères culturelles }\end{array}$ & 1975 & VIII $1-2$ & $85-100$ \\
\hline $\begin{array}{l}\text { DUFOUR, Marie-France } \\
\text { Nouvelles avenues considérées par le } \\
\text { service de probation }\end{array}$ & 1979 & XII 2 & $41-57$ \\
\hline $\begin{array}{l}\text { DUMONT, Hélène } \\
\text { Parthenais; début d'une lutte }\end{array}$ & 1976 & IX $1-2$ & $163-190$ \\
\hline $\begin{array}{l}\text { ÉLIE, Daniel } \\
\text { La criminalité au Québec }\end{array}$ & 1981 & XIV I & $85-104$ \\
\hline $\begin{array}{l}\text { ELLENBERGER, Henri } F \text {. } \\
\text { Mutilations corporelles infligées aux } \\
\text { femmes : étude victimologique }\end{array}$ & 1980 & XIII 1 & $80-93$ \\
\hline $\begin{array}{l}\text { EUGÈNE, Carl } \\
\text { La gestion de cas en libération condi- } \\
\text { tionnelle }\end{array}$ & 1981 & XIV 2 & $25-40$ \\
\hline $\begin{array}{l}\text { EVANS, John L. } \\
\text { Le programme de recherches sur la } \\
\text { victimisation au Canada }\end{array}$ & 1980 & XIII 1 & $104-106$ \\
\hline $\begin{array}{l}\text { FATTAH, Ezzat } \\
\text { Victimologie : tendances récentes }\end{array}$ & 1980 & XIII 1 & $6-36$ \\
\hline $\begin{array}{l}\text { FAUGERON, Claude } \\
\text { Représentations du système de justice } \\
\text { criminelle : essai de typologie }\end{array}$ & 1973 & VI & $13-66$ \\
\hline
\end{tabular}




\section{FORTIER, Julien}

Une expérience pilote de sentence de travaux communautaires

FRECHETTE, Marcel

Le criminel et l'autre : analyse des relations interpersonnelles de l'homme criminel

Délinquance, socialisation et névrosisme

Le comité Paul Rose

\section{GAGNE, Denis}

Interprétation psycho-culturelles de l'inadaptation juvénile dans la société de masse contemporaine

GAGNON, Astrid

Parthenais; début d'une lutte

GAGNON, Rosette

Les praticiens de la criminologie au Québec

GALLANT, Gérald

Une expérience pilote de sentence de travaux communautaires

GAUTHIER, Maurice

Le processus décisionnel de libération conditionnelle

GIROUX, Jocelyn

Le citoyen et la justice

GOYER-MICHAUD, Francyne

Vers une nouvelle conception des valeurs : apport de la psychologie à la criminologie des valeurs

L'éducatrice : une sur-femme?

GRENIER, Hélène

Les normes de l'intervention auprès des jeunes mésadaptés - Bilan des écrits

Les petits commerçants victimes de vol à main armée en quête de justice

HETU, Jean

Le pauvre, la machine judiciaire et la détention

HUOT, Louis

Le citoyen et la justice
Année Vol./No. Pages

$1979 \quad$ XII $2 \quad 24-40$

$1970 \quad$ III $\quad 11-102$

$1975 \quad$ VIII 1-2 53-84

1981 XIV $2 \quad 95-98$

1968 I $\quad 9-134$

$1976 \quad$ IX 1-2 163-190

$1977 \quad \times 2 \quad 39-54$

$1979 \quad$ XII $2 \quad 24-40$

$1981 \quad$ XIV $2 \quad 61-72$

$1979 \quad$ XII $1 \quad 43-56$

$1973 \quad$ V1 67.146

1975 VIII l-2 159-166

$1978 \quad$ XI $2 \quad 42-75$

$1979 \quad$ XII $1 \quad 57-65$

1976 IX 1-2 87-106

$1979 \quad$ XII $1 \quad 43-56$ 


\begin{tabular}{lrr} 
Année & Vol./No. & Pages \\
\cline { 3 - 4 } 1979 & XII 2 & $41-57$ \\
1976 & IX 1-2 & $5-22$ \\
1969 & II & $145-168$ \\
1970 & III & $103-142$ \\
1981 & XIV I & $31-50$ \\
1971 & VI & $11-112$
\end{tabular}

quent youth: some sociocultural considerations

LABERGE-ALTMEJD, Danielle Les normes de l'in tervention auprès des jeunes mésadaptés - bilan des écrits

LACOSTE, Michel

Nouvelles avenues considérées par le service de probation

LANDREVILLE, Pierre

L'application des règles minima pour le traitement des détenus au Canada

$\begin{array}{ccc}1973 & \text { VI } & 147-198 \\ 1976 & \text { IX 1-2 } & 107-117 \\ 1976 & \text { IX 1-2 } & 5-22 \\ 1981 & \text { XIV 2 } & 95-98 \\ & & \\ 1978 & \text { XI 2 } & 76-87 \\ 1979 & \text { XII 1 } & 78-80 \\ 1979 & \text { XII 2 } & 78-88\end{array}$
que criminologique

Les détenus et les droits de l'homme

Les origines de la prison de Bordeaux

Le comité Paul Rose

LANGELIER, Gilles

Vers l'établissement de normes de qualité de services dans les centres d'accueil du Québec

LAPLANTE, Jacques

Le citoyen face à la déviance 


\begin{tabular}{|c|c|c|}
\hline Année & Vol. $/$ No. & Pages \\
\hline 1968 & I & $9-135$ \\
\hline 1970 & III & $171-207$ \\
\hline 1971 & IV & $113-192$ \\
\hline 1972 & V & $107-182$ \\
\hline $\begin{array}{l}1974 \\
1975\end{array}$ & $\begin{array}{c}\text { VII } \\
\text { VIII } 1-2\end{array}$ & $\begin{array}{l}107-132 \\
101-118\end{array}$ \\
\hline 1975 & VIII $1-2$ & $145-158$ \\
\hline 1978 & XI 1 & $24-45$ \\
\hline 1978 & XI J & $7-23$ \\
\hline
\end{tabular}

LEGER, Gérald J.

Le programme de recherches sur la victimisation au Canada

$1980 \quad$ XIII $1 \quad 104-106$

\section{LEMIRE, Guy}

La libération conditionnelle : le point de vue de la sociologie

$1981 \quad$ XIV $2 \quad 81-94$

\section{LIPPE, Michel}

La protection de la jeunesse et le projet de loi 65

1975 VIII 1-2 9-28

\section{LOUIS-GUERIN, Christiane}

Réflexions méthodologiques pour une recherche sur l'opinion publique et l'action sociale : essai de synthèse

1979 XII 1

$24-42$

MANSEAU, Hélène

Les petits commerçants victimes de vol à main armée en quête de justice

1979

XII $1 \quad 57-65$

MARINEAU, Daniel

La dimension cachée : introduction à des programmes de rééducation en milieu libre

MARQUIS, Jacques

Le crime économique

1977

X 1

79-93

MENARD, Robert

Le climat social dans les institutions pour jeunes délinquants 


\section{MORIN-LAURENDEAU, Nicole}

Une expérience de court terme selon Carkhuff au service de probation du Québec

NICOLAS, Michel

Un rappel historique de la libération conditionnelle : deux volets d'une évolution

NORMANDEAU, André

Towards the measurement of criminality in Canada : a replication study Synthèse des travaux : ler symposium international de recherche en criminologie comparée

Violence and robbery : A case study

Les prisons de l'an 1984

Les praticiens de la criminologie au Québec

Dialogue avec André Normandeau propos recueillis par G. Paradis

Le public et la violence; l'analyse des rapports des commissions d'enquête

Faut-il transformer ou abolir la probation?

Le vol à main armée

Le comité Paul Rose

PARADIS, Georges

Dialogue avec André Normandeau propos recueillis par G. Paradis

PARIZEAU, Alice

Les droits de la jeunesse et le projet de loi C-192

Sommes-nous tous des assasins?

Délinquance juvénile et société

Les droits des mineurs et l'emprisonnement des jeunes au Québec

Le crime organisé et les pactes de nonagression. Réflexions du juge Robert Cliche recueillies par A. Parizeau

Le clan des Dubois

L'Association Mondiale des Amis de l'Enfance et les problèmes de la structuration du bénévolat

De l'esprit et de l'application des normes

Des théories génétiques aux normes de traitement; l'analyse d'un livre d'Yves

Roumajon

Le dédommagement des victimes

L'armée et la Crise d'octobre

\begin{tabular}{ccc} 
Année & Vol. No. & Pages \\
\cline { 3 - 4 } 1979 & & \\
& & \\
& & \\
& & \\
1981 & XIV 2 & $73-80$ \\
& & \\
1968 & I & $135-260$ \\
& & \\
1970 & III & $143-170$ \\
1972 & V & $11-106$ \\
1976 & IX 1-2 & $232-238$ \\
1977 & X 2 & $39-54$ \\
1977 & X 2 & $78-92$ \\
1979 & XII 1 & $81-88$ \\
1979 & XII 2 & $89-100$ \\
1981 & XIV 1 & $105-110$ \\
1981 & XIV 2 & $95-98$ \\
& & \\
1977 & X 2 & $78-92$ \\
& &
\end{tabular}




\section{PRECOURT, André}

Les praticiens parlent... La surveillance en libération conditionnelle, plaidoyer pour un changement d'orientation

\section{RACICOT, Jacques}

Les praticiens parlent... La surveillance en libération conditionnelle, plaidoyer pour un changement d'orientation

\section{RAHAV, Giora}

The measurement of movements on the conformity-deviance continuum as an auxiliary tool for action-research

RICO, José $M$.

L'indemnisation des victimes d'actes criminels : étude comparative

Commissions d'enquête sur la justice pénale au Canada

La prison dans la Chine de Mao

Notes introductives à l'étude de la criminalité des affaires

La société de criminologie du Québec : historique, activités et perspectives d'avenir

Les événements d'octobre 1970 et l'administration de la justice pénale au Québec

RIVARD, Paul-André

Le service de probation du Québec : évolution et rôles traditionnels

\section{RIZKALLA, Samir}

Les recherches sur la police: trois approches

Crime and criminal justice in the developing countries

L'Association professionnelle des criminologues du Québec, dix ans après

ROBERT, Philippe

Représentations du système de justice criminelle : essai de typologie

SARRASIN, Marie

La gestion de cas en libération conditionnelle

\section{Année \\ Vol./No. Pages}

1981

XIV 2

7-24

$1981 \quad$ XIV $2 \quad 7.24$

1970

III

103-142

1968

I

261-312

1971

IV

209-219

1976

IX $1-2$

219-231

1977

X 1

8-28

1977

X 2

55-61

1980

XIII 2

7-46

1979

XII 2

$7-23$

1972

v

183-197

1974

VII

169-191

1977

$\mathrm{X} 2$

62-78

1973

VI

13-66

1981

XIV $225-40$ 
SHOHAM, Shlomo

The measurement of movements on the conformity-deviance continuum as an auxiliary tool for action-research

SWEENAY, Bernard

La prison et la responsabilité collective

$\begin{array}{lcc}\text { Année } & \text { Vol./No. } & \text { Pages } \\ 1970 & \text { III } & 103-142 \\ 1976 & \text { IX 1-2 } & 151-162\end{array}$

SZABO, Denis

Interprétation psycho-culturelles de l'inadaptation juvénile dans la société de masse contemporaine

Synthèse des travaux : ler symposium international de recherche en criminologie comparée

La nouvelle criminologie et la délinquance

Histoire d'une expérience québécoise qui aurait pu mal tourner

Société, culture et criminalité : essai sur les limites de l'interprétation étiologique et praxéologique

$\begin{array}{ccc}1968 & \text { I } & 9-134 \\ 1970 & \text { III } & 143-170 \\ 1975 & \text { VIII 1-2 } & 175-188 \\ 1977 & \text { X 2 } & 5-38 \\ 1981 & \text { XIV 1 } & 7-30 \\ 1978 & \text { XI 1 } & 24-45 \\ 1974 & \text { VII } & 107-132\end{array}$

TESSIER, Bernard

Les étapes de la rééducation : formalisation et vérification

THI-HAU, Nguyen

Réactions sociales à la déviance : une étude exploratoire

THIREAU, Louise

La gestion de cas en libération conditionnelle

$1981 \quad$ XIV $2 \quad 25-40$

TREMBLAY, Reneault

Le contrôle informel des gérants de banques et caisses populaires lors d'infractions bancaires

$1977 \quad X 1 \quad 46-68$

TREMBLAY, Richard E.

Une question d'actualité : l'intégration scolaire dans les centres d'accueil : impact sur les objectifs poursuivis et le climat social

$1978 \quad$ XI $1 \quad 65-74$

TROTTIER, Michel-Jacques

Nouvelles avenues considérées par le service de probation

$1979 \quad$ XII $2 \quad 41-57$

WALLER, Irvin

La criminalité au Canada et aux Etats-Unis: tendances et explications comparatives (1964-1978) 
WATSON, G.A.B.

Critique de la justice criminelle

Année

Vol./No. Pages

YOSHIMASU, Shufu

Etudes criminologiques et psychiatriques au Japon

1969

II

$145-168$

ZUPANCIC, Bostjan

Criminal Law and its influence upon normative integration

1974

VII

53-106 


\section{II \\ INDEX DES SUJETS}

\section{ATTITUDE DU PUBLIC}

Violence et répression : mythe ou réalité?

Micheline BARIL

Le rôle et l'utilité du public dans l'élaboration des politiques criminelles Yves BRILLON

Le citoyen et la justice

\section{Louis HUOT et}

Jocelyn GIROUX

Le citoyen face à la déviance

$$
\text { Jacques LAPLANTE }
$$

Réactions sociales à la déviance : une étude exploratoire

Marc LEBLANC et

Nguyen THI-HAU

Réflexions méthodologiques pour une recherche sur l'opinion publique et l'action sociale : essai de synthèse Christiane LOUIS-GUERIN

Les petits commerçants victimes de vol à main armée en quête de justice Hélène MANSEAU et Hélène GRENIER

Le public et la violence; l'analyse des rapports des commissions d'enquête André NORMANDEAU

1979

XII 1

$66-77$

1979 XII 1

1979

XII 1

43-56

1979

XII 1

$78-80$

1974

VII

$107-132$

1979

XII 1

24-42

1979

XII 1

57.65

1979

XII 1

$81-88$

Représentations du système de justice criminelle : essai de typologie

Philippe ROBERT et

Claude FAUGERON

\section{BIBLIOGRAPHIES}

Bibliographie sélective sur la rééducation

$$
\text { Vesna BLAZINA }
$$

Littérature carcérale québécoise

$$
\text { Lise BRUNET-AUBRY }
$$

Bibliographie générale Jacqueline DE PLAEN

Information bibliographique Jacqueline DE PLAEN

Information bibliograhique Jacqueline DE PLAEN

$\begin{array}{ccc}1978 & \text { XI 1 } & 80-86 \\ 1976 & \text { IX 1-2 } & 191-195 \\ 1979 & \text { XII 2 } & 101-105 \\ 1980 & \text { XIII 1 } & 107-108 \\ 1981 & \text { XIV 1 } & 111-118\end{array}$


Aperçu bibliographique des travaux de l'Université de Montréal, 19601978

Jacqueline DE PLAEN et Aniela CZETWERTYNSKA

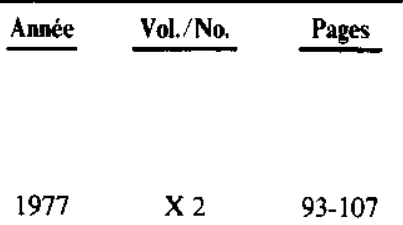

COMMISSION D'ENQUETE

La crise d'octobre et les commissions

d'enquête Jean-Paul BRODEUR

Le public et la violence; l'analyse des rapports et des commissions d'enquête André NORMANDEAU

Le vol à main armée André NORMANDEAU

Commissions d'enquêtes sur la justice pénale au Canada

José M. RICO

\section{COUT DU CRIME}

The cost of crime and of social defence against crime : Summary of the second International Symposium in comparative criminology

\section{CRIME ECONOMIQUE}

La pègre, un milieu d'affaires Jean-Pierre CHARBONNEAU

Le crime organisé et les pactes de nonagression. Réflexions du juge Cliche recueillies par A. Parizeau

Robert CLICHE

Le crime économique

$$
\text { Jacques MARQUIS }
$$

Notes introductives à l'étude de la criminalité des affaires

José M. RICO

1977

$\begin{array}{ll}X & 8-28\end{array}$

Le contrôle informel des gérants de banques et caisses populaires lors d'infractions bancaires

$$
\text { Reneault TREMBLAY }
$$

\section{CRIME ORGANISE}

La pègre, un milieu d'affaires Jean-Pierre CHARBONNEAU

Le crime organisé et les pactes de nonagression. Réflexions du juge Cliche recueillies par $\mathrm{A}$. Parizeau

\section{Robert CLICHE et}




\section{CRIMINOLOGIE COMPAREE}

Comparative criminology : Africa Suzanne ARCAND et Yves BRILLON

La transe vaudouesque : un syndrome de déviance socio-culturelle Emerson DOUYON

La famille et la délinquance dans trois sphères culturelles

Emerson DOUYON

Criminalité et répression de la criminalité au Japon : analyse socio-culturelle et criminologie

Hans-Heiner KÜHNE

Synthèse des travaux : ler symposium international de recherche en criminologie comparée

André NORMANDEAU et

Denis SZABO

Crime and criminal justice in the developing countries

Samir RIZKALLA

The measurement of movements on the conformity-deviance continuum as an auxiliary tool for action-research

Shlomo SHOHAM,

Giora RAHAV et

A. KREIZLER

Société, culture et criminalité : essai sur les limites de l'interprétation étiologique et praxéologique Denis SZABO

La criminalité au Canada et aux États-Unis : tendances et explications comparatives (1964-1978)

Irvin WALLER

Etudes criminologiques et psychiatriques au Japon

Shufu YOSHIMASU et

Sadataka KOGI

CRIMINOLOGUES ET CRIMINO-

\section{LOGIES}

Aperçu bibliographique des travaux de l'Université de Montréal, 19601978

Jacqueline DE PLAEN et Aniela CZETWERTYNSKA
Année

Vol./No.

Pages

1973

VI

199-217

1969

II

$11-70$

1975

VIII 1.2

$85-100$

1981

XIV 1

$31-50$

1970

III

143-170

1974

VII

169-191

1970

III

103-142

$1981 \quad$ XIV I

$7-30$

1981

XIV 1

$51-84$

1969

II

$145-168$ 
Année

Vol./No. Pages

Les patriciens de la criminologie au Québec

Rosette GAGNON et

André NORMANDEAU

1977

$\mathrm{X} 2$

$39-54$

Dialogue avec André Normandeau propos recueillis par G. Paradis

André NORMANDEAU et

Georges PARADIS

1977

$\times 2$

$78-92$

La société de criminologie du Québec : historique, activités et perspectives d'avenir

José M. RICO

1977

$\times 2$

$55-61$

L'Association professionnelle des criminologues du Québec, dix ans après

Samir RIZKALLA

1977

$X 2$

$62-64$

Histoire d'une expérience québécoise qui aurait pu mal tourner

Denis SZABO

$\mathrm{X} 2 \quad 5-38$

\section{DÉLINQUANCE FÉMININE}

Self-image and delinquency : a contribution to the study of female criminality and woman's image

Marie-Andrée BERTRAND

1969

II $\quad 7 !-144$

DÉVIANCE

La transe vaudouesque : un syndrome de déviance psycho-culturelle

Emerson DOUYON

1969

II $\quad 11-70$

Le citoyen face à la déviance Jacques LAPLANTE

XII $1 \quad 78-80$

Réactions sociales à la déviance : une étude exploratoire

Marc LEBLANC et

Nguyen THI-HAU

The measurement of movements on the conformity-deviance continuum as an auxiliary tool for action-research

Shlomo SHOHAM,

Giora RAHAV and

A. KREIZLER

1970

II]

103-142

DROITS DES DETENUS

Parthenais; début d'une lutte Astrid GAGNON et Hélène DUMONT

$163-190$

L'application des règles minima pour le traitement des détenus au Canada

Pierre LANDREVILLE 


\section{Les détenus et les droits de l'homme Pierre LANDREVILLE}

\section{DROITS DES MINEURS}

La protection de la jeunesse et le Projet de loi 65

$$
\text { Michel LIPPÉ }
$$

Les droits de la jeunesse et le projet de loi C-192

$$
\text { Alice PARIZEAU }
$$

Sommes-nous tous des assasins?

$$
\text { Alice PARIZEAU }
$$

Les droits des mineurs et l'emprisonnement des jeunes au Québec

$$
\text { Alice PARIZEAU }
$$

La prison et la responsabilité collective

$$
\text { Bernard SWEENAY }
$$

ÉTIOLOGIE DE LA DELINQUANCE

La réaction sociale à la délinquance juvénile : une analyse stigmatique

$$
\text { Marc LEBLANC }
$$

La délinquance en milieu aisé : un groupe culture d'adeptes de la drogue

\section{Marc LEBLANC}

La prison et la responsabilité collective

\section{Bernard SWEENAY}

Interprétations psycho-culturelles de l'inadaptation juvénile dans la société de masse contemporaine
Denis SZABO
Marc LEBLANC
Lise DESLAURIERS et
Denis GAGNÉ

\section{LEGISLATIONS DES MINEURS}

La protection de la jeunesse et le projet de loi 65

$$
\text { Michel LIPPE }
$$

Les droits de la jeunesse et le projet de loi C-192

$$
\text { Alice PARIZEAU }
$$

\section{LIBERATION CONDITIONNELLE}

La libération conditionnelle : le point de vue des acteurs

$$
\text { Carmen ARSENAULT }
$$

Année

Vol./No.

Pages

1976

IX 1-2

$107-117$

197

$$
\text { VIII 1-2 } \quad 9-28
$$

1975 VIII 1-2 29-52

$1975 \quad$ VIII $1-2 \quad 167.174$

$1976 \quad$ IX $1-2 \quad 118-150$

1976

IX 1-2 151-162

1971

IV

113-192

1972

$\mathrm{V}$

$107-182$

1976

IX $1-2 \quad 151-162$

1968

9-134

1975

VIII 1-2

$9-28$

1975

VIII 1-2

29-52

1981

XIV 2

41-60 
Le comité Paul Rose

Année Vol./No. Pages

Pierre CARRIËRE

Marcel FRECHETTE

Pierre LANDREVILLE et

André NORMANDEAU

$1981 \quad$ XIV $2 \quad 95-98$

La gestion de cas en libération conditionnelle

Carl EUGENE

Marie SARRASIN et

Louise THIREAU

$1981 \quad$ XIV $2 \quad 25-40$

Le processus décisionnel de libération conditionnelle

Maurice GAUTHIER

La libération conditionnelle : le point de vue de la sociologie

Guy LEMIRE

Un rappel historique de la libération conditionnelle : deux volets d'une évolution

Michel NICOLAS

$981 \quad$ XIV $2 \quad 73-80$

Les praticiens parlent... La surveillance en libération conditionnelle, plaidoyer pour un changement d'orientation

André PRECOURT et Jacques RACICOT

$1981 \quad$ XIV $2 \quad 7-24$

Normes

voir DROITS DES DÉTENUS

POLITIQUE CRIMINELLE

TRAITEMENT DES

JEUNES DÉLINQUANTS

NOTES DE LECTURE

Surveiller et punir Jean-Paul BRODEUR

Littérature carcérale québécoise Lise BRUNET-AUBRY

$1976 \quad$ IX 1-2 196-218

1976 IX 1-2 191-195

Les normes de l'intervention auprès des jeunes mésadaptés - Bilan des écrits

Maurice CUSSON

Danielle LABERGE-ALTMEJD et Hélène GRENIER

$1978 \quad$ XI $2 \quad 42.75$

La crise, telle qu'on l'a écrite Jacqueline DE PLAEN

$1980 \quad$ XIII $2 \quad 99-110$

Le programme de recherches sur la victimisation au Canada

Gérald J. LEGER et

John L. EVANS

1980 XIII l 104-106 
Les prisons de l'an 1984 André NORMANDIN

\section{Année}

1976

IX 1-2

$232-238$

Le public et la violence; l'analyse des rapports des commissions d'enquête

André NORMANDEAU

Faut-il transformer ou abolir la probation?

André NORMANDEAU

Délinquance juvénile et société

Alice PARIZEAU

Le clan des Dubois

\section{Alice PARIZEAU}

L'Association mondiale des amis de l'enfance et les problèmes de la structuration du bénévolat

Alice PARIZEAU

Des théories génétiques aux normes de traitement; l'analyse d'un livre d'Yves Roumajon

Alice PARIZEAU

La prison dans la Chine de Mao José M. RICO

La nouvelle criminologie et la délinquance

Denis SZABO

\section{PEINE}

Les prisons du Québec; ghet to des pauvres

André CAMPEAU

Deux modalités de la peine et leurs effets sur le criminel

Maurice CUSSON

Une expérience pilote de sentence de travaux communautaires

\section{Julien FORTIER et}

Gérald GALLANT

Le pauvre, la machine judiciaire et la détention

Jean HETU

POLICE

Les recherches sur la police : trois approches

Samir RIZKALLA

POLITIQUE CRIMINELLE

Le rôle et l'utilité du public dans l'élaboration des politiques criminelles Yves BRILLON
1972

V

183-197

1979

XII 1

81-88

1979

XII 2

89-100

1975 VIII 1-2 189-194

$1977 \times 1$

94-97

1978

XI 1

$75-79$

$1978 \quad$ XI 2

88-93

1976

IX 1-2

219-231

1975

VIII 1-2

175-188

1976

IX 1-2

$60-86$

1974

VII

11-52

1979

XII 2

$24-40$

1976

IX 1-2

$87-106$

1979

XII 1

6-23 


\section{Les normes de l'intervention auprès
des jeunes mésadaptés - Bilan des écrits \\ Maurice CUSSON \\ Danielle LABERGE-ALTMEJD et Hélène GRENIER \\ Vers l'établissement de normes de qualité de services dans les centres d'accueil du Québec}

Année

Vol./No. Pages

Gilles LANGELIER

1978

XI 2

76-87

De l'esprit et de l'application des normes

Alice PARIZEAU

1978

XI 2

Critique de la justice criminelle

G.A.B. WATSON

1978

$\mathrm{XI} 2$

6-24

\section{PRISONS ET PÉNITENCIERS}

La prison de Bordeaux et sa population
D. DELISLE

1976

IX 1-2

23-59

Parthenais; début d'une lutte

Astrid GAGNON et

Hélène DUMONT

1976

IX 1-2 163-190

Les origines de la prison de Bordeaux

Pierre LANDREVILLE et

Ghislaine JULIEN

197

IX $1-2 \quad 5-22$

Les prisons de l'an 1984

\section{André NORMANDEAU}

1976

IX $1-2 \quad 232-238$

La prison et la responsabilité collective

Bernard SWEENAY

1976

IX 1-2 151-162

\section{PROBATION}

La surveillance de la liberté et la pratique criminologique

Yvon DANDURAND et

Jacques LAPLANTE

1979

XII $2 \quad 78-88$

Bibliographie générale Jacqueline DE PLAEN

La probation pour mineurs au Québec Marc LEBLANC

1975 VIII $1-2 \quad 101.118$

Une expérience de court terme selon Carkhuff au service de probation du Québec

$$
\text { Nicole MORIN-LAURENDEAU }
$$

197

XII $2 \quad 58-77$

Faut-il transformer ou abolir la probation? 


\section{SOCIOLOGIE DU DROIT}

Criminal law and its influence upon normative integration

Bostjan ZUPANCIC

\section{STATISTIQUES CRIMINELLES}

Towards the measurement of criminality in Canada : a replication study

Doyan D. AKMAN et André NORMANDEAU

La prison de Bordeaux et sa population

D. DELISLE

La criminalité au Québec Daniel ÉLIE

La délinquance d'hier et de demain au Québec

Marc LEBLANC

La criminalité au Canada et aux États-Unis: tendances et explications comparatives (1964-1978)

Irvin WALLER

TERRORISME

La crise d'octobre et les commissions d'enquête Jean-Paul BRODEUR

La crise, telle qu'on l'a écrite Jacqueline DE PLAEN

L'armée et la crise d'octobre Alice PARIZEAU

Les événements d'octobre 1970 et l'administration de la justice pénale au Québec

José M. RICO

\section{TOXICOMANIE}

La délinquance en milieu aisé : un groupe culture d'adeptes de la drogue

Marc LEBLANC

1972

1980

XIII 2

79-98

1980

1980

XIII 2

XIII $2 \quad 47.78$

1980

XIII 2

$7-46$

TRAITEMENTS DES JEUNES

\section{DELINQUANTS}

Bibliographie sélective sur la rééducation

Vesna BLAZINA

1978

XI 1

$80-86$

Traitement des jeunes délinquants : modèle d'appariement selon le niveau conceptuel

Ronald BRILL 


\begin{tabular}{|c|c|c|c|}
\hline & Année & Vol./No. & Pages \\
\hline $\begin{array}{l}\text { L'observation du comportement des } \\
\text { jeunes en institution } \\
\text { Maurice CUSSON }\end{array}$ & 1975 & VIII $1-2$ & $119-144$ \\
\hline $\begin{array}{l}\text { Les normes de l'intervention auprès } \\
\text { des jeunes mésadaptés - bilan des } \\
\text { écrits } \\
\text { Maurice CUSSON } \\
\text { Danielle LABERGE-ALTMEJD et } \\
\text { Hélène GRENIER }\end{array}$ & 1978 & XI 2 & 42.75 \\
\hline $\begin{array}{l}\text { L'éducatrice : une sur-femme? } \\
\text { Francyne GOYER-MICHAUD }\end{array}$ & 1975 & VIII $1-2$ & $159-166$ \\
\hline $\begin{array}{l}\text { Treatment and rehabilitation of delin- } \\
\text { quent youth : some sociocultural } \\
\text { considerations } \\
\text { Lenore KUPPERSTEIN }\end{array}$ & 1971 & VI 2 & $11-112$ \\
\hline $\begin{array}{l}\text { Vers l'établissement de normes de } \\
\text { qualité de services dans les centres } \\
\text { d'accueil du Québec } \\
\text { Gilles LANGELIER }\end{array}$ & 1978 & XI 2 & $76-87$ \\
\hline $\begin{array}{l}\text { La probation pour mineurs au Québec } \\
\text { Marc LEBLANC }\end{array}$ & 1975 & VIII 1-2 & $101-118$ \\
\hline $\begin{array}{l}\text { Les étapes de la rééducation : formali- } \\
\text { sation et vérification } \\
\text { Marc LEBLANC et } \\
\text { Bernard TESSIER }\end{array}$ & 1978 & XI 1 & $24-45$ \\
\hline $\begin{array}{l}\text { La dimension cachée : introduction à } \\
\text { des programmes de rééducation en mi- } \\
\text { lieu libre } \\
\text { Daniel MARINEAU }\end{array}$ & 1974 & VII & 133-168 \\
\hline $\begin{array}{l}\text { Le climat social dans les institutions } \\
\text { pour jeunes dėlinquants } \\
\text { Robert MÉNARD et } \\
\text { Marc LEBLANC }\end{array}$ & 1978 & XI 1 & 7.23 \\
\hline $\begin{array}{l}\text { Une question d'actualité : l'intégra- } \\
\text { tion scolaire dans les centres } \\
\text { d'accueil : impact sur les objectifs } \\
\text { poursuivis et le climat social } \\
\text { Richard E. TREMBLAY }\end{array}$ & 1978 & XI 1 & $65-74$ \\
\hline $\begin{array}{l}\text { VICTIMOLOGIE } \\
\text { Violence et répression : mythe ou réa- } \\
\text { lité? } \\
\text { Micheline BARIL }\end{array}$ & 1979 & XII l & $66-77$ \\
\hline $\begin{array}{l}\text { Ils n'ont plus la liberté : réactions à la } \\
\text { victimisation et ses conséquences } \\
\text { Micheline BARIL }\end{array}$ & 1980 & XIII 1 & $94-103$ \\
\hline $\begin{array}{l}\text { La victimologie et le viol, un discours } \\
\text { complice } \\
\text { Renée COLETTE-CARRIERE }\end{array}$ & 1980 & XIII 1 & $60-79$ \\
\hline
\end{tabular}




\begin{tabular}{|c|c|c|c|}
\hline & Année & Vol./No. & Pages \\
\hline $\begin{array}{l}\text { Information bibliographique } \\
\text { Jacqueline DE PLAEN }\end{array}$ & 1980 & XIII 1 & $107-108$ \\
\hline $\begin{array}{l}\text { Mutilation corporelles infligées aux } \\
\text { femmes : étude victimologique } \\
\text { Henri F. ELLENBERGER }\end{array}$ & 1980 & XIII 1 & $80-93$ \\
\hline $\begin{array}{l}\text { Victimologie : tendances récentes } \\
\text { Ezzat FATTAH }\end{array}$ & 1980 & XIII l & $6-36$ \\
\hline $\begin{array}{l}\text { Le citoyen et la justice } \\
\text { Louis HUOT et } \\
\text { Jocelyn GIROUX }\end{array}$ & 1979 & XII l & $43-56$ \\
\hline $\begin{array}{l}\text { Le programme de recherches sur la } \\
\text { victimisation au Canada } \\
\text { Gérald J. LEGER et } \\
\text { John L. EVANS }\end{array}$ & 1980 & XIII 1 & $104-106$ \\
\hline $\begin{array}{l}\text { Les petits commerçants victimes de } \\
\text { vol à main armée en quête de justice } \\
\text { Hélène MANSEAU et } \\
\text { Hélène GRENIER } \\
\text { Le dédommagement des victimes } \\
\text { Alice PARIZEAU }\end{array}$ & 1980 & XIII I & $\begin{array}{l}57-65 \\
37-59\end{array}$ \\
\hline $\begin{array}{l}\text { VIOL } \\
\text { La victimologie et le viol, un discours } \\
\text { complice } \\
\text { Renée COLETTE-CARRIERE }\end{array}$ & 1980 & XIII I & 60.79 \\
\hline $\begin{array}{l}\text { VOL } \\
\text { Violence and robbery : A case study } \\
\text { André NORMANDEAU }\end{array}$ & 1972 & V & $11-106$ \\
\hline $\begin{array}{l}\text { Le vol à main armée } \\
\text { André NORMANDEAU }\end{array}$ & 1981 & XIV 1 & $105-110$ \\
\hline
\end{tabular}

